\title{
Determination of the interaction potential from the pair distribution function: An inverse Monte Carlo technique
}

\author{
N. G. Almarza and E. Lomba \\ Instituto de Química Física, Rocasolano (CSIC), C/ Serrano 119, E-28006 Madrid, Spain
}

(Received 15 November 2002; published 3 July 2003)

\begin{abstract}
In this work we present an efficient procedure to evaluate effective pair potentials compatible with "experimental" distribution functions using a Monte Carlo simulation scheme. Using computer simulation results for the pair distribution functions, we have applied the method to a Lennard-Jones fluid and to a model of liquid aluminum. In both cases the procedure was able to recover with high accuracy the actual interaction potential of the systems. Moreover, the procedure can easily incorporate additional information, for instance, thermodynamic properties, in order to improve the reliability of the results.
\end{abstract}

DOI: 10.1103/PhysRevE.68.011202 PACS number(s): 61.20.Ja, 05.20.Gg, 02.70.-c, 61.20.Qg

\section{INTRODUCTION}

The inverse problem, i.e., the determination of the Hamiltonian of a given system from information on its microscopic structure, remains one of the key problems in the physics of many-particle systems.

The available direct experimental information on the structure of condensed phases reduces basically to the structure factor $S(q)$, which is nothing but the Fourier transform of the pair distribution function $g(r)$. This being a pair function, the simplest approach would then be the construction of an effective pair potential that would render the same pair structure as that of the system under consideration. The uniqueness theorem due to Henderson [1] guarantees that if a pair potential can be found, which reproduces $g(r)$ of the system, then this potential is unique. Note, however, that this does not guarantee the existence of such a pair potential. In addition, $g(r)$ must be known with very high precision over all values of $r$ in order to expect that the uniqueness theorem guarantees a reliable result for the effective potential. Starting from the work of Johnson, Hutchinson, and March [2] various works have attempted to use theoretical approaches to solve the inverse problem with varying range of success [3] culminating in the relatively recent work of Kahl, Bildstein and Rosenfeld [4] where an integral equation based on Rosenfeld's principle of universality of the bridge functional [5] was constructed and successfully applied to binary mixtures of simple fluids. Despite this apparent success, methods solely based on the use of theoretical approaches have a very limited applicability. Even the method of Kahl et al. [4] cannot be expected to overcome the limitations of the universality principle of the bridge functional, i.e., systems that deviate substantially from the packing geometry of the hard sphere fluid will hardly be amenable to be tackled within this approach. Moreover, the use of theoretical approximations for systems other than simple fluids would be computationally hampered by the difficulties associated with the treatment of the orientational degrees of freedom.

A more promising, though computationally more demanding, approach was introduced by Levesque, Weis, and Reatto [6], who proposed the combination of an integral equationpredictor step—with computer simulation-corrector step - to generate a pair potential compatible with a given input $g(r)$. The weakness of the method lies in the need to extract the bridge function from the simulation data, a process plagued with numerical inaccuracies. This function is then inserted in a modified hypernetted chain (MHNC) equation that is inverted to predict a new estimate of the pair potential. Alternatively one can resort to simulation in the predictor and corrector step, as suggested by Dzugutov [7], which avoids the use of the MHNC extrapolation scheme at the cost of using extremely large samples in the simulation stage.

More recently Soper [8] has proposed a simulation-based iterative procedure where a test pair potential is modified using the difference between the test model potential of mean force and the one corresponding to the system under consideration. Each iteration implies a complete equilibration of the simulation sample using the test potential before the next correction is applied. This procedure, known as empirical potential structure refinement (EPSR), has been successfully applied to analyze the experimental structure of a variety of systems [9]. Despite its success, the method, as currently formulated does not correctly account for the thermodynamic properties, even when the input $g(r)$ corresponds to a strictly pairwise additive potential energy [8]. We are well aware that the ability to capture the structure and thermodynamics of a given system by means of effective pair interactions is limited by the relative weight of the $n$-body $(n \geqslant 3)$ potential energy contributions to the internal energy. However, if in the system under scrutiny only the pair terms of the potential energy are relevant, one should demand that the solution of the inverse problem for the structure be also a solution for the thermodynamics.

Recently a rather sophisticated approach has been proposed by Lyubartsev and Laaksonen [10] and has been applied to the determination of effective interionic pair potentials in electrolytes, an application of relevance for this type of technique that enables the reformulation of a hardly tractable problem in computationally feasible terms. The recipe of Lyubartsev and Laaksonen requires the solution of a set of linear equations and relatively long simulations with the trial pair potential in each refinement step.

A somewhat different approach is adopted in the original formulation of the reverse Monte Carlo (RMC) method [11]. 
Here, one simply generates Monte Carlo moves that minimize the difference between the test pair distribution function (or structure factor) and the input reference $g(r)$ [or $S(q)$ ], using a $\chi^{2}$ statistics. This $\chi^{2}$ plays the role of the potential energy in a standard canonical Monte Carlo method. The procedure thus formulated does not generate a Hamiltonian and, strictly speaking, is not a solution of the inverse problem. Nonetheless, it provides information on the microscopic structure of the system compatible with a given pair structure function. Obviously, the standard RMC method, its newer variants [12], and its molecular dynamics (MD) analog [13] are not constrained by the use of effective pair potentials, but they have, however, a tendency to predict structures with the maximum configurational disorder compatible with the input $g(r)$ [14]. This will not be necessarily so in methods where the energy is constrained, like those based on the use of effective pair potentials [9].

The scenario depicted above clearly indicates that there is still room for improvement. In this paper we propose an inverse Monte Carlo approach for the determination of effective pair potentials, essentially free from the limitations that affect the methods commented upon in the previous paragraphs. Our method is inspired by Wang and Landau's procedure [15] to evaluate the density of states in lattice models. We will see that the procedure converges rapidly and the refinement steps can be performed along the simulation run without requiring a complete equilibration run every step. This considerably reduces the computational effort as compared to other simulation-based procedures. Moreover, the method is amenable to incorporate information on system properties in addition to the pair structure, and thus we will show that it is possible to reproduce simultaneously the internal energy and the microscopic structure of a given system without significantly increasing the computational effort. This obviously applies only to systems that can be described entirely in terms of pair interactions. On the other hand, if information on the three-body structure is also available our procedure could be extended to determine simultaneously two- and three-body effective interactions without requiring essential modifications of the computational scheme. This situation certainly can hardly apply to the analysis of experimental data, where information on three particle correlations is only indirectly available, but it might be of interest when obtaining effective potentials to perform simulations of complex biomolecules or organic molecules whose interactions can be reproduced with difficulty by simple pair interactions.

We will illustrate the efficiency of the proposed procedure solving the inverse problem for a simple Lennard-Jones (LJ) system and for a classical model for liquid aluminum due to Dagens, Rasolt, and Taylor (DRT) [16], the same two systems investigated by Levesque et al. [6]. The case of liquid $\mathrm{Al}$, whose interionic potential exhibits a very characteristic structure, is particularly illustrative of the capabilities of this inverse Monte Carlo approach. In this case, the estimated effective potential, once appropriately smoothed, will be used in a MD calculation and it will be shown that at least some of the dynamic properties of the original DRT model are also recovered.

The rest of the paper is organized as follows. The essen- tials of the inverse Monte Carlo method are introduced in Sec. II and are illustrated with its application to the recovery of a simple LJ potential. The relation between structure and thermodynamics and the combined use of both in the inverse simulation is analyzed in Sec. III, where it is exemplified for the DRT model of liquid Al. Finally, in Sec. IV we present and discuss our most significant results.

\section{METHOD}

We are interested in deriving an effective pair potential $v(r)$, which for given conditions of temperature $T$ and density $\rho$ will correspond to a known radial distribution function (RDF) $g(r)$. In what follows we will sketch a numerical procedure that makes use of the ideas underlying the method recently proposed by Wang and Landau [15] to evaluate the density of states of lattice models. The method proposed here can be applied to models of different complexity. As an example, it will be shown how it can be used to determine the effective potential of a monoatomic fluid.

To some extent, our procedure and those of Levesque, Weis, and Reatto [6], Soper [8], and Lyubartsev and Laaksonen [10] approach the inverse problem on a similar footing, namely, the use of trial interaction potentials that are improved iteratively by comparing their corresponding RDF's with that of the "real" system. In our method, however, the basic idea is to perform simulations in which the pair interaction can change along the run (without waiting for the simulation to provide good statistics for a fixed trial potential). These changes in the trial potential are tuned in order to achieve the convergence of the RDF of the simulated system toward the given input $g(r)$. The essential input data to run the procedure are the number of particles $N$, the density $\rho$, and the "target" RDF $g(r)$ of the system under study. The procedure is organized in several stages; at each stage we expect to be closer to the best effective potential and therefore the corrections will be progressively smaller, so that at the final stages the running effective potential will be practically constant, and will provide the expected RDF.

\section{A. The scheme in action}

Let us suppose that the RDF $g(r)$ and its error bars $\Delta g(r)$ of a certain fluid are known, with data tabulated as discrete points: $\left(r_{i}, g_{i}, \Delta g_{i}\right)$ with $i=0,1, \ldots, m-1$, and $r_{i}=r_{0}+(i$ $+1 / 2) \delta r$. We will assume $g(r)=0$, for $r<r_{0}$ [that is, $\beta v(r)=\infty$ for $r<r_{0}$ and $\beta v(r)=0$ for distances greater than those tabulated for $g(r)]$, where $v(r)$ represents the effective pair potential and $\beta=1 / k_{B} T$. We will estimate numerically the value of the interaction for the discrete points $r_{i}$ and use the values $\beta v_{i}=\beta v\left(r_{i}\right)$ to interpolate the effective interaction, $\beta v(r)$ at any given distance $r$, using the two nearest values of $r_{i}$.

In order to start the procedure we need an initial guess for the effective interaction and a particle configuration. In our case we have chosen to use $\beta v\left(r_{i}\right)=-\ln g\left(r_{i}\right)$ and a fcc lattice structure [17]. Then we can start the simulation procedure at the first stage $(l=1)$. At each stage $l$ and after each cycle (or fixed number of cycles) of $N$ trial displacements, 
we compute the pair distances to evaluate a global RDF of the stage $g_{l}(r)$ and the instantaneous RDF $g_{\text {inst }}(r)$ (corresponding to the current configuration of the system), and modify $\beta v(r)$ from the current values $\beta v^{\text {old }}$ to the new estimates $\beta v^{\text {new }}$ using

$$
\begin{gathered}
\beta v_{i}^{\text {new }}=\beta v_{i}^{\text {old }}+\lambda_{l} \frac{g_{\text {inst }}\left(r_{i}\right)-g_{i}}{\Delta g_{i}}\left\langle\Delta g_{i}\right\rangle, \\
\left\langle\Delta g_{i}\right\rangle=\frac{1}{m} \sum_{i=0}^{m-1} \Delta g_{i} .
\end{gathered}
$$

After each block of $n_{c}$ cycles of simulation we check the convergence criterion,

$$
\frac{1}{m} \sum_{i=0}^{m-1}\left(\frac{g_{l}\left(r_{i}\right)-g_{i}}{\Delta g_{i}}\right)^{2} \leqslant \eta
$$

A sensible value of the convergence parameter in condition (3) is $\eta \simeq 1$. If condition (3) is fulfilled, we start a new stage $l+1$ and modify the value of $\lambda$ as

$$
\lambda_{l+1}=\alpha \lambda_{l}=\alpha^{l-1} \lambda_{1},
$$

with $0<\alpha<1$. The fulfillment of condition (3) at a certain stage does not guarantee that we have reached the correct result of $\beta v(r)$. For relatively large values of $\lambda$ we are not performing an equilibrium simulation, since the interaction potential used along the run is not constant. In practical terms, however, the main features of the interaction potential appear already at the very first stages of the procedure, and then the result is steadily refined and the numerical noise is reduced in the subsequent stages. The end of the refinement process can be established by comparing the differences between effective potentials at the end of two successive stages.

\section{B. Application}

As a first example, we have considered a fluid of spherical particles with pair interactions defined through a truncated and shifted $[17,18]$ Lennard-Jones potential with a cutoff distance $r_{c}=2.5 \sigma$, where $\sigma$ is the interaction diameter. For such a model we have evaluated the RDF $g_{L J}(r)$ and estimated the corresponding error bars using MC simulation $[17,18]$ in the canonical ensemble $(N V T)$ of a system of 500 particles at reduced density $\rho \sigma^{3}=0.80$ and reduced temperature $k_{B} T / \epsilon=1.0$. The width of the intervals to measure $g_{L J}$ was $\delta r / \sigma=0.01$. The RDF was evaluated using $3.2 \times 10^{4}$ cycles after equilibration. The larger values of $\Delta g_{L J}\left(r_{i}\right)$ (estimated as the standard deviation of the mean for the results of blocks of $4 \times 10^{3}$ cycles) were about $1 \times 10^{-3}$.

With the $g_{L J}(r)$ values extracted as indicated above we start the inverse simulation procedure, using the same number of particles and volume that in the direct calculation, with $\lambda_{1}=0.10, \alpha=0.50, \eta=1$, and a number of stages $n_{l}$ $=17$. The convergence criterion was found to be satisfied after a number of cycles around $4 \times 10^{3}$ for the first stages (excluding stage 1), whereas for the last stages the simula-

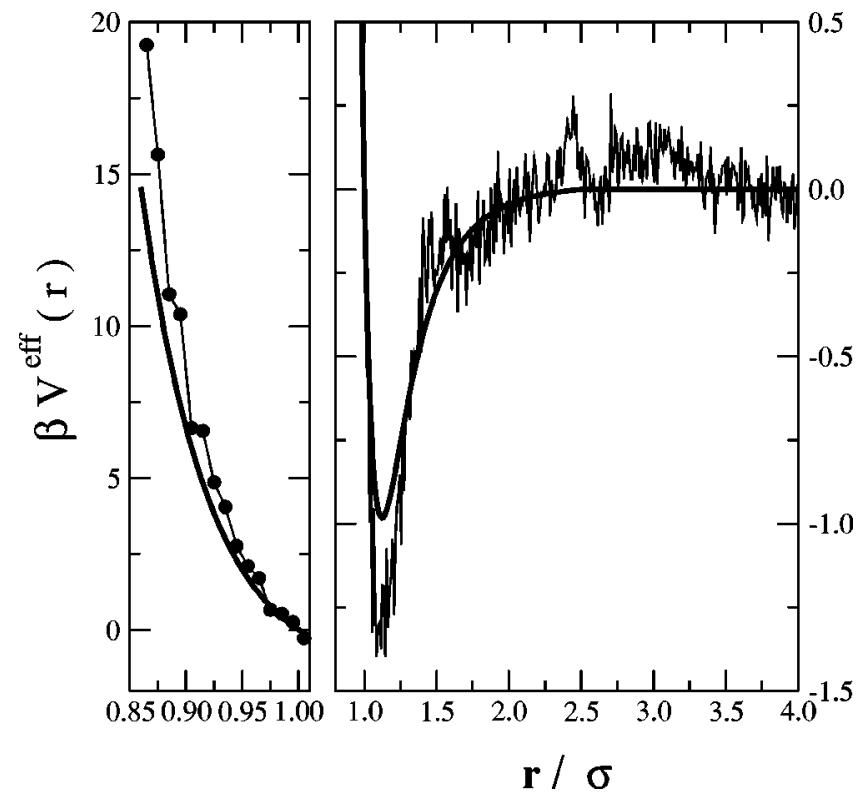

FIG. 1. Real potential (continuous thick line) and effective potential (continuous thin line and filled circles) extracted after the completion of the first stage of the inversion procedure for the Lennard-Jones test case.

tion lengths were about twice that of the direct calculation.

In Fig. 1 we show the result of the effective potential after completion of the first stage of the procedure, while in Fig. 2 the results at the end of the last stage are presented. From Fig. 1 it seems clear that even at the end of the first stage the inversion procedure has captured the essential features of the interaction potential. The small differences between the "real" potential and that obtained after the completion of the inverse procedure (see Fig. 2) are mainly due to the uncer-

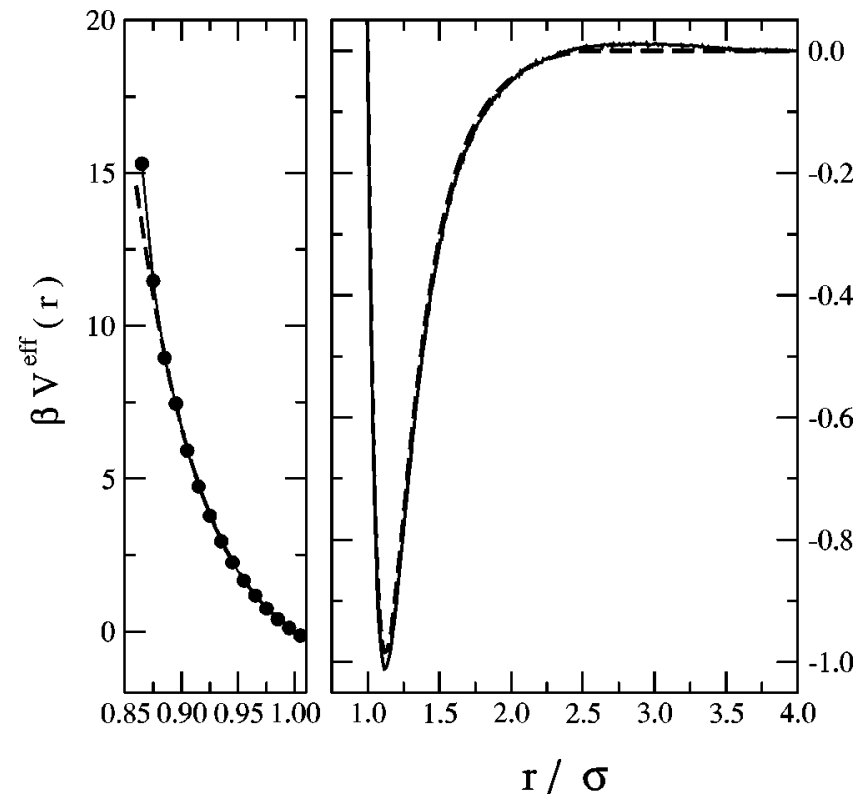

FIG. 2. Real potential (dashed line) and effective potential (continuous line and filled circles) extracted from the inversion method for the Lennard-Jones test case. 
tainties in $g_{L J}(r)$. In fact, moderate uncertainties in $g_{L J}(r)$ can lead to a broad distribution of effective pair potentials compatible with the RDF within a given convergence parameter $\eta$. If such is the case, one can choose smaller values of $\eta$ in order to obtain "better" estimates of $\beta v_{\text {eff }}$. An estimation of the uncertainty of $\beta v_{\text {eff }}$ can be done by running the inversion procedure using $\eta=1$ from different initial conditions (random number sequence, starting guess for the effective potential, different values of $\lambda$, etc.) and analyzing the dispersion of the numerical results of $\beta v_{\text {eff }}$. In any case, one should not expect to find a very precise determination of the effective interaction by using a very demanding convergence criterion in condition (3) if the experimental RDF is not extremely precise.

\section{STRUCTURE AND THERMODYNAMICS}

At this point it is important to recall that the precision of the experimental RDFs of real systems is usually not very high. It can, therefore, be desirable to combine the structural information with other experimental data (e.g., thermodynamic properties) to, hopefully, improve the quality of the effective interaction estimations. As pointed out above, a wide set of acceptable solutions for the effective potential can be found when the RDF is not very precise. It is then possible to search for solutions also compatible with the experimental values of certain thermodynamic properties (for instance, the internal energy or the pressure). In any case there must be a clear connection between the additional properties and the RDF. In what follows we will explain how these ideas can be put forward to include the potential energy value in the inversion procedure (the pressure could also be included with similar techniques).

The potential energy per particle, $u_{\text {inst }}$, of a given configuration of a simulated system, can be written in terms of the effective potential, for a system with spherical interactions as

$$
\frac{u_{\text {inst }}}{k_{B} T} \simeq \frac{\rho}{2} \int_{r_{0}}^{r_{c}} d \mathbf{r} g_{\text {inst }}(r) \beta v_{\text {eff }}(r),
$$

where $r_{0}$ and $r_{c}$ are, respectively, the lower and upper limits of RDF tabulation. In order to secure the convergence of the average of $u_{\text {inst }}$ to its experimental value $u$, we can perform small corrections of the effective potential by shifting uniformly $\beta v_{\text {eff }}(r)$. Our choice was

$$
\delta \beta v_{\text {shift }}=\frac{2}{\rho V_{\text {int }}}\left(\beta u-\beta u_{\text {inst }}\right) \lambda_{u, l},
$$

where

$$
V_{i n t}=\frac{4 \pi}{3}\left(r_{c}^{3}-r_{0}^{3}\right)
$$

and $\lambda_{u, l}$ depends on the stage of the calculation in the same way as described in Eq. (4) for $\lambda_{l}$,

$$
\lambda_{u, l}=\alpha \lambda_{u, l-1}=\alpha^{l-1} \lambda_{u, 1} .
$$

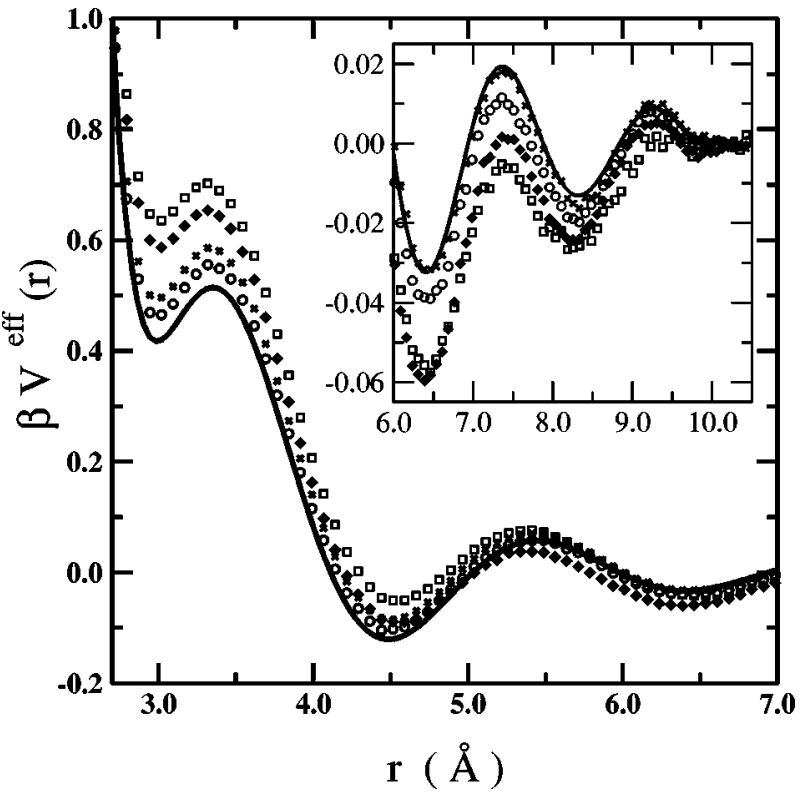

FIG. 3. Results for the aluminum model. The DRT pair potential $\beta v(r)$ is presented with continuous line. The effective potentials extracted using the inversion procedure with different convergence criteria are represented with symbols $A$ (squares), $B$ (filled diamonds), $C$ (crosses), and $D$ (circles).

In this way, we can use the following equation to update the running effective potential:

$$
\beta v_{i}^{\text {new }}=\beta v_{i}^{\text {old }}+\lambda_{l} \frac{g_{\text {inst }}\left(r_{i}\right)-g_{i}}{\Delta g_{i}}\left\langle\Delta g_{i}\right\rangle+\delta \beta v_{\text {shift }} .
$$

Within this scheme we must include an additional convergence criterion

$$
\frac{\left|u_{l}-u\right|}{\Delta u} \leqslant \eta_{u}
$$

where $u_{l}$ is the average of the energy of the system at stage $l, \Delta u$ is the error bar of the experimental potential energy, and $\eta_{u}$ is the energy convergence parameter.

\section{Application on a model potential of aluminum}

We have checked the performance of both procedures, with and without energetic considerations, on a model potential of liquid aluminum $[6,16]$, which exhibits more complex features than the Lennard-Jones interaction (see Fig. 3). In this case the RDF was evaluated using a molecular dynamics simulation on a $N V T$ ensemble [19] with $N=500$ at a number density $\rho=0.0527 \AA^{-3}$ and $T=1051 \mathrm{~K}$. We performed $2 \times 10^{5}$ MD steps with a time step of 2.5 fs. The system was considered equilibrated after the first $2 \times 10^{3}$ steps and averages were performed every ten time steps. The interaction potential was truncated at $r_{c}=9.78 \AA$, and the RDF was evaluated up to a distance of $10.58 \AA$ (which corresponds to half the length of the simulation box) with a grid of $\delta r$ $\simeq 0.025 \AA$. The larger values of $\Delta g\left(r_{i}\right)$ for this system were about 0.002 . 
The inverse simulation procedure was applied using four different convergence requirements. In all the cases we used $\lambda_{1}=0.5, \alpha=0.5$, and $n_{l}=14$. The first case $(A)$ implies no energy optimization and a convergence parameter $\eta=1.0$, the second one $(B)$ includes energy optimization with $\eta$ $=1.0, \eta_{u}=1.0$, and $\lambda_{u, 1}=1.0$. Finally, the third case $(C)$ runs without energy optimization using $\eta=0.10$ and the fourth case $(D)$ uses $\eta=0.10$ and energy optimization with $\eta_{u}=0.1$. In cases $(B)$ and $(D)$ the energy converges to its input value $u / k_{B} T=5.66$, whereas in $(A)$ it converges toward $u / k_{B} T=4.417$ and in $(C)$ toward $u / k_{B} T=5.18$. One sees immediately that an stringent convergence criterion in the structure implies also a considerable improvement in the thermodynamics.

The results of the effective potential obtained are shown in Fig. 3. In the four cases the inversion procedure captures the main features of the interaction potential; however, it seems clear from the figure that even using a precise input for the RDF the dispersion of the effective interactions statistically compatible with $g(r)$ is not negligible. The inversion procedure using a stronger convergence criterion provides the results with the best agreement with the actual interaction potential. In addition, it is observed that the improvement of the quality of the effective potential carried out by considering the energy optimization procedure is not very important. Of course, in the case of real systems the accuracy of the thermodynamic properties that can be incorporated in the inversion procedure is usually much higher than that of the structural data. Such a situation will probably enhance the effects of taking into account thermodynamic data as an additional input. Moreover, if we are interested in using the effective potential to gain further insight in the behavior of the real system by means of molecular modeling, the introduction of thermodynamic optimization can become crucial in quite a number of applications.

A final check of the procedure was done using the effective potentials of cases $C$ and $D$ after an appropriate cubic spline smoothing in two MD runs and comparing the results obtained with those of the DRT model. The results for the internal energy were in total agreement with those of the MC calculations reported above. As to the pressure, the MD runs yielded a pressure of $121.1 \pm 2 \mathrm{kbar}$ for case $C$ and 115.4 \pm 2 for case $D$, the latter in complete agreement with the original DRT model result $115.5 \pm 2 \mathrm{kbar}$. Also, the DRT model diffusion constant $6.1 \times 10^{-9} \mathrm{~m}^{2} / \mathrm{s}$ is in accordance with the values obtained using the potential of cases $C$ and $D, 6.2 \times 10^{-9} \mathrm{~m}^{2}$ and $5.7 \times 10^{-9} \mathrm{~m}^{2}$, respectively. The fact that the dynamics of the DRT model is to some extent well captured by these effective potentials is further supported by inspection of the vibrational density of states plotted in Fig. 4. One immediately sees that the differences between the original potential and those resulting from the inverse procedure are hardly noticeable. Perhaps other properties such as the dynamic structure factor might reflect more openly the differences between the models $C$ and $D$ and the original DRT model.

\section{FINAL REMARKS AND CONCLUSIONS}

From our experience in the present work, it seems that the choice $\alpha=0.5$ works fine. In the selection of the value of $\lambda_{1}$

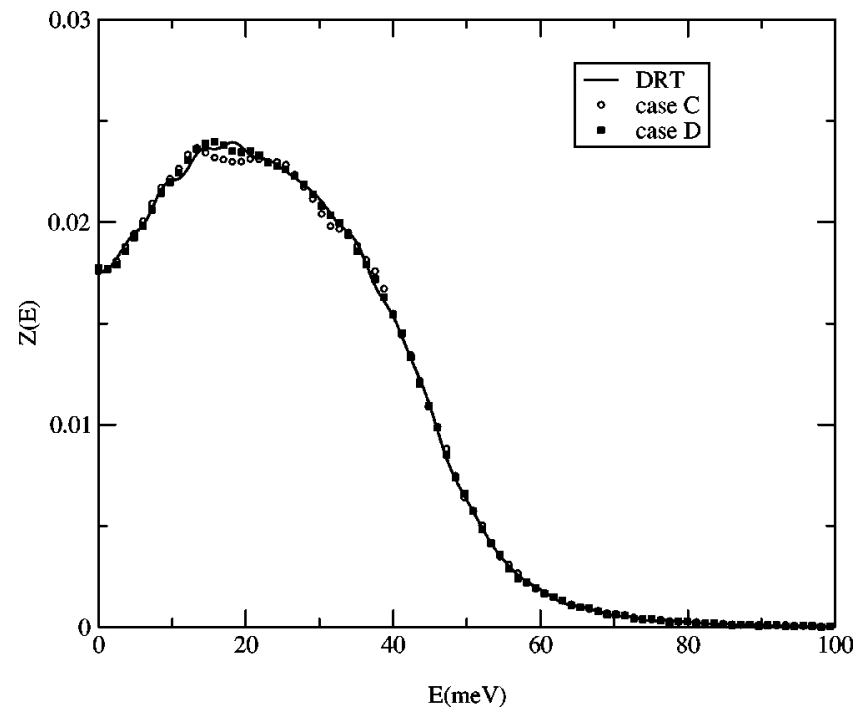

FIG. 4. Vibrational density of states for the DRT model of liquid $\mathrm{Al}$ (solid curve) and obtained using the effective potentials of cases $C$ and $D$ (symbols).

two facts are to be borne in mind: a large value of $\lambda_{1}$ enhances the convergence of the first stage, but it also introduces a high level of noise in $\beta v(r)$ that will have to be smeared out in the subsequent stages, which can increase the length of the corresponding runs. In order to save computing time, further refinements can be introduced in the method, for instance, one may incorporate an equilibration period at the beginning of the first stage without starting the accumulation of data in $g_{1}(r)$ (in order to reduce the effects of the initial conditions on the number of cycles required to fulfill the convergence criterion). It may also be possible to smooth the current values of the effective potential before starting a new stage (in order to reduce the effects of the statistical noise introduced by the stochastic nature of the method).

We have checked the convergence behavior of the method with respect to the initial values of $\beta v_{i}$ by starting the simulation with a hard-sphere potential instead of the potential of mean force. No substantial influence on the convergence rates was observed. Incidentally, during the process of this work we have seen that the procedure can also be useful to check the correctness of computer simulation programs.

Following a similar approach to that used in the introduction of the energy optimization it could also be possible to find an efficient procedure to use the structure factor instead of the pair distribution function as experimental input of the inversion procedure.

In summary, we have presented a general method to determine effective interaction potentials from the information provided by distribution functions. Our method is simpler to use than those previously reported in the literature, and takes into account implicitly the different uncertainties of the values of $g(r)$ for different distances. The method has not shown any convergence problems for the systems considered in this paper. It has been applied to atomic fluids, but it can be easily generalized, for instance, to mixtures of spherical particles or to fluids of rigid molecules. The accuracy of the results depends on the quality of the RDF data; therefore, 
since the experimental data of real systems are typically not very precise, it could be worthy to consider the possibility of complementing the inversion procedure with other available information on the experimental system (for instance, thermodynamic properties); this extra information can be seemlessly introduced in the framework of the procedure.

As a final remark, it must be stressed that the ability of these effective pair potentials derived using an inverse method to reproduce experimental properties-other than those explicitly used as input in the inversion procedure-is strongly dependent on the availability of high precision structural data, and on the relative weight of many-body contributions to the potential energy.

\section{ACKNOWLEDGMENTS}

The authors acknowledge the financial support of the Dirección General de Investigación Científica under Grant Nos. MAT2002-04540-C05-03/05 (N.G.A.) and BFM20011017-C03-01 (E.L.).
[1] R.L. Henderson, Phys. Lett. 49A, 197 (1974).

[2] M.D. Johnson, P. Hutchinson, and N.H. March, Proc. R. Soc. London, Ser. A 282, 283 (1964).

[3] W.S. Howells and J.E. Enderby, J. Phys. C 5, 1277 (1972); N.K. Ailawadi, P.K. Banerjee, and A. Choudy, J. Chem. Phys. 60, 2571 (1974); M.W. Dharma-Wardana and G.C. Aers, Phys. Rev. Lett. 56, 1211 (1986).

[4] G. Kahl, B. Bildstein, and Y. Rosenfeld, Phys. Rev. E 54, 5391 (1996).

[5] Y. Rosenfeld, J. Chem. Phys. 98, 8126 (1993), and references therein.

[6] D. Levesque, J.J. Weis, and L. Reatto, Phys. Rev. Lett. 54, 451 (1985).

[7] M. Dzugutov, Phys. Rev. A 40, 5434 (1989).

[8] A.K. Soper, Chem. Phys. 202, 295 (1996).

[9] T. Yamaguchi, K. Hidaka, and A.K. Soper, Mol. Phys. 96, 1159 (1999).

[10] A.P. Lyubartsev and A. Laaksonen, Phys. Rev. E 52, 3730 (1995).

[11] R.L. McGreevy and L. Pusztai, Mol. Simul. 1, 369 (1988).
[12] F.L. da Silva, B. Svensson, T. Åkeson, and B. Jönson, J. Chem. Phys. 109, 2624 (1998).

[13] G. Toth and A. Baranyai, J. Chem. Phys. 114, 2027 (2001).

[14] P. Jedlovsky, I. Bako, G. Palinkas, and A.K. Soper, J. Chem. Phys. 105, 245 (1996).

[15] F. Wang and D.P. Landau, Phys. Rev. Lett. 86, 2050 (2001); Phys. Rev. E 64, 056101 (2001).

[16] L. Dagens, M. Rasolt, and R. Taylor, Phys. Rev. B 11, 2726 (1975).

[17] M. P. Allen and D. J. Tildesley, Computer Simulation of Liquids (Clarendon Press, Oxford, 1986).

[18] D. Frenkel and B. Smit, Understanding Molecular Simulation (Academic Press, New York, 1996).

[19] All MD simulations presented in this work have been carried out using the MD package DLPOLY 2.13. This is a package of molecular simulation routines written by W. Smith and T. Forester. Copyright: The Engineering Council of Physical Sciences Research Council, acting through its Daresbury and Rutherford Appleton Laboratories at Daresbury Laboratory, 1994. 